\title{
Bayesian experimental design without posterior calculations: an adversarial approach Supplementary material
}

\author{
Dennis Prangle* Sophie Harbisher ${ }^{\dagger}$ and Colin S Gillespie ${ }^{\dagger}$ \\ dennis.prangle@bristol.ac.uk s.harbisher@newcastle.ac.uk \\ colin.gillespie@newcastle.ac.uk
}

\section{A Scoring rule details}

Section 3.2 of the main paper describes scoring rules and related concepts. Here, Table 1 summarises two strictly proper scoring rules of particular interest in this paper: logarithmic score and Hyvärinen score (Hyvärinen, 2005). It also gives their associated entropies and divergences. The Hyvärinen results rely on the following regularity conditions:

H1. $p(\theta)$ and $q(\theta)$ are twice differentiable with respect to all $\theta_{i}$.

H2. $\mathbb{E}_{\theta \sim p(\theta)}\left[\|\nabla \log p(\theta)\|^{2}\right], \mathbb{E}_{\theta \sim q(\theta)}\left[\|\nabla \log q(\theta)\|^{2}\right]$ are finite.

H3. $\nabla p(\theta) \rightarrow 0, \nabla q(\theta) \rightarrow 0$ for $\|\theta\| \rightarrow \infty$.

It this section we use $\nabla$ without a subscript to represents gradient with respect to $\theta$. Also recall that $\|\theta\|$ represents the $L_{2}$ norm. Finally, note that several of the results of this paper use the following assumption.

A5 Conditions H1-H3 hold for $p(\theta)=\pi(\theta \mid y ; \tau)$ and $q(\theta)=\pi(\theta)$, given any $y, \tau$.

\begin{tabular}{lll}
\hline & Logarithmic score & Hyvärinen score \\
\hline Scoring rule & $-\log q(\theta)$ & $2 \Delta \log q(\theta)+\|\nabla \log q(\theta)\|^{2}$ \\
Entropy & $-\mathbb{E}_{\theta \sim p(\theta)}[\log p(\theta)]$ & $-\mathbb{E}_{\theta \sim p(\theta)}\left[\|\nabla \log p(\theta)\|^{2}\right]$ \\
Divergence & $\mathbb{E}_{\theta \sim p(\theta)}[\log p(\theta)-\log q(\theta)]$ & $\mathbb{E}_{\theta \sim p(\theta)}\left[\|\nabla \log p(\theta)-\nabla \log q(\theta)\|^{2}\right]$ \\
\hline
\end{tabular}

Table 1: Summary of two scoring rules and related quantities. Here $p(\theta)$ is the true density of an unobserved quantity and $q(\theta)$ is a generic density. Note that $\Delta$ is the Laplacian operator (sum of second partial derivatives) with respect to $\theta$. For a derivation of the Hyvärinen divergence see Appendix A of Hyvärinen (2005). The other derivations are straightforward.

\section{B Framework summaries}

Table 2 summarises and contrasts the decision-theoretic and game-theoretic frameworks from Sections 3 and 4.

\footnotetext{
* School of Mathematics, University of Bristol, UK

$\dagger$ School of Mathematics Statistics and Physics, Newcastle University, UK
} 


\section{Bayesian experimental design without posterior calculations: supplement}

\begin{tabular}{l|l} 
Decision theoretic & Game theoretic \\
\hline - E selects design $\tau$ & - E selects design $\tau$ \\
& - C selects matrix $A$ \\
- $\mathbf{N}$ generates parameters $\theta$ from prior & - N generates parameters $\theta$ from prior \\
(unobserved until reward allocated) & (unobserved until reward allocated) \\
- N generates data $y$ from model & - N generates data $y$ from model \\
- E selects $a$, estimated density for $\theta$ & - E selects $a$, estimated density for $\phi=A^{-1} \theta$ \\
- E receives reward $\mathcal{R}(a, \theta)$ & - E receives reward $\mathcal{R}(a, \phi)$ \\
& - C receives reward $-\mathcal{R}(a, \phi)$ \\
\hline
\end{tabular}

Table 2: Two frameworks for experimental design: the approach of Bernardo (1979) (left), and our modification (right), with changes shown in blue. The abbreviations are for Experimenter, Critic and Nature. Players observe all actions except $\theta$.

\section{Proofs}

\section{C.1 Proof of Result 1}

We will show the following which suffices to give the required result. As in the statement of Result 1, all expectations are with respect to $\pi(\theta, y ; \tau)$.

1. The expected reward from design $\tau$ equals $\mathbb{E}\left[\mathcal{U}_{\text {entropy }}\right]$, assuming the experimenter selects $a$ to maximise their expected utility.

2. $\mathbb{E}\left[\mathcal{U}_{\text {entropy diff }}\right]$ and $\mathbb{E}\left[\mathcal{U}_{\text {divergence }}\right]$ also equal this value up to an additive constant.

Proofs of both parts are below. The first is a consequence of A1. The proof of the second part is based on the common observation that the divergence contains a constant term which can be ignored under maximisation. Similar results have appeared in the experimental design literature previously for a logarithmic score function (e.g. Shewry and Wynn, 1987). This result simply generalises them to a general score function.

Note that manipulations of expectations used below are valid by A2 and Fubini's theorem.

Part 1 First fix some values of $\tau$ and $y$ and consider the experimenter's choice of $a$. This must maximise the expected reward, which is, using A1,

$$
-\mathbb{E}_{\theta \sim \pi(\theta \mid y ; \tau)}[\mathcal{S}(a, \theta)] .
$$

Since $\mathcal{S}$ is a strictly proper scoring rule, the optimal choice of $a$ is the posterior $\pi(\theta \mid y ; \tau)$. The resulting expected reward is $\mathcal{U}_{\text {entropy }}(\tau, y)$. (We write $\mathcal{U}_{\text {entropy }}$ having only the arguments $\tau, y$ as it does not depend on $\theta$.)

Now suppose $y$ is randomly generated by nature. Then the expected reward of design $\tau$ is the expectation of $\mathcal{U}_{\text {entropy }}(\tau, y)$ with respect to $f(y \mid \theta, \tau)$, or equivalently with respect to $\pi(\theta, y ; \tau)=\pi(\theta) f(y \mid \theta ; \tau)$, as required. 
Part 2 From our definitions,

$$
\mathcal{U}_{\text {divergence }}=\mathcal{U}_{\text {entropy }}+\mathbb{E}_{\theta \sim \pi(\theta \mid y ; \tau)}[S(\pi(\theta), \theta)] .
$$

We are interested in expectations of utility with respect to $\pi(\theta, y ; \tau)$, which equals $\pi(\theta \mid y ; \tau) \pi(y ; \tau)$ by $(3)$. Thus

$$
\begin{aligned}
\mathbb{E}_{(\theta, y) \sim \pi(\theta, y ; \tau)}\left[\mathcal{U}_{\text {divergence }}\right] & =\mathbb{E}_{(\theta, y) \sim \pi(\theta, y ; \tau)}\left[\mathcal{U}_{\text {entropy }}\right]+\mathbb{E}_{(\theta, y) \sim \pi(\theta, y ; \tau)}[S(\pi(\theta), \theta)] \\
& =\mathbb{E}_{(\theta, y) \sim \pi(\theta, y ; \tau)}\left[\mathcal{U}_{\text {entropy }}\right]+\mathcal{H}[\pi(\theta)] \\
& =\mathbb{E}_{(\theta, y) \sim \pi(\theta, y ; \tau)}\left[\mathcal{U}_{\text {entropy diff }] .}\right.
\end{aligned}
$$

(Note A2 guarantees the terms above are finite.)

So $\mathcal{U}_{\text {divergence }}$ and $\mathcal{U}_{\text {entropy diff }}$ produce the same expected utility. Furthermore the expected utility of $\mathcal{U}_{\text {entropy }}$ only differs by a finite additive constant, $\mathcal{H}[\pi(\theta)]$.

\section{C.2 Proof of Result 2}

It's sufficient to show that these utilities have the same expectation as $\mathcal{U}_{\text {divergence }}$ with respect to $\pi(\theta, y ; \tau)$. From Table 1 (and using A5 for the Hyvärinen score case):

$$
\mathcal{U}_{\text {divergence }}= \begin{cases}\mathbb{E}_{\theta \sim \pi(\theta \mid y ; \tau)}[\log \pi(\theta \mid y ; \tau)-\log \pi(\theta)] & \text { (log score) } \\ \mathbb{E}_{\theta \sim \pi(\theta \mid y ; \tau)}\left[\left\|\nabla_{\theta} \log \pi(\theta \mid y ; \tau)-\nabla_{\theta} \log \pi(\theta)\right\|^{2}\right] & \text { (Hyvärinen score) }\end{cases}
$$

The quantities within the expectations are $\mathcal{U}_{\text {SIG }}$ and $\mathcal{U}_{\text {FIG }}$ respectively. Thus these have same expectations as $\mathcal{U}_{\text {divergence }}$ under $\pi(\theta, y ; \tau)$. Finally note that from $(4), \mathcal{U}_{\text {trace }}=$ $\mathbb{E}_{y \sim f(y \mid \theta ; \tau)}\left[\mathcal{U}_{\mathrm{FIG}}\right]$. So this has the same expectation under $\pi(\theta, y ; \tau)$ as $\mathcal{U}_{\mathrm{FIG}}$. (These manipulations of expectations are valid using Fubini's theorem and assumption A2 for the log score case, or A5 for the Hyvärinen score case.)

\section{C.3 Proof of Result 3}

As the logarithmic score is a proper scoring rule, $a(\tau, A, y)$ must output the posterior. Using the change of variables formula, the posterior for $\phi=A^{-1} \theta$ is

$$
\pi_{\Phi}(\phi \mid y ; \tau)=\pi_{\Theta}(\theta \mid y ; \tau)|\operatorname{det} A|,
$$

where $\pi_{\Theta}(\theta \mid y ; \tau)$ is the posterior for $\theta$. Using A3, $\operatorname{det} A=1$ so $\pi_{\Phi}(\phi \mid y ; \tau)=\pi_{\Theta}(\theta \mid y ; \tau)$. Hence the expected reward to the experimenter from $\tau$ given $a(\tau, A, y)$ is, regardless of the choice of $A, \mathbb{E}_{\theta, y \sim \pi(\theta, y ; \tau)}\left[-\log \pi_{\Theta}(\theta \mid y ; \tau)\right]$, the same as in the decision theoretic framework. Thus $\tau$ must optimise the same objective in the decision theoretic setting, or in a SPE of the game theoretic setting.

\section{C.4 Proof of Result 4}

Let "game 1" be based on the original parameters $\theta$, and "game 2" use the alternative parameters $B \theta$. 


\section{Bayesian experimental design without posterior calculations: supplement}

\section{Part 1: Relation between action sequences}

Here we show that there are invertible mappings $A_{2}=f\left(A_{1}\right), a_{2}=g\left(a_{1}\right)$ such that actions $\left(\tau, A_{1}, y, a_{1}\right)$ in game 1 , and $\left(\tau, A_{2}, y, a_{2}\right)$ in game 2 have the same expected rewards up to a multiplicative constant that depends only on $B$. In game $1, a_{1}$ is a density $a_{1}\left(\phi_{1}\right)$ for $\phi_{1}=A_{1}^{-1} \theta$. The expected reward to the experimenter is then $r_{1}=-\mathbb{E}_{\theta \sim \pi(\theta \mid y ; \tau)}\left[\mathcal{S}\left(a_{1}, A_{1}^{-1} \theta\right)\right]$ where $\mathcal{S}$ is Hyvärinen score.

First suppose $\operatorname{det} B>0$. In this case let $A_{2}=k^{-1 / p} B A_{1}$ where $k=\operatorname{det} B$ and $p=\operatorname{dim}(\theta)$. This is a valid action in game 2 since $\operatorname{det} A_{2}=1$. In game 2 the experimenter must select a density on $\phi_{2}=A_{2}^{-1} B \theta=k^{1 / p} \phi_{1}$. Let $a_{2}\left(\phi_{2}\right)=a_{1}\left(k^{-1 / p} \phi_{2}\right) / k$. Now consider the actions $\left(\tau, A_{2}, y, a_{2}\right)$ in game 2 . The expected reward to the experimenter is then $r_{2}=-\mathbb{E}_{\theta \sim \pi(\theta \mid y ; \tau)}\left[\mathcal{S}\left(a_{2}, k^{1 / p} A_{1}^{-1} \theta\right)\right]$. From the definition of Hyvärinen score, $r_{2}=k^{-2 / p} r_{1}$. (As reparameterising by a scalar preserves Hyvärinen score up to proportionality.)

Now suppose $\operatorname{det} B<0$. (Recall $B$ is invertible so $\operatorname{det} B \neq 0$.) Let $F$ be a $p \times p$ identity matrix with the top left entry changed to -1 . Now let $A_{2}=k^{-1 / p} B A_{1} F$ where $k=$ $|\operatorname{det} B|$. Again det $A_{2}=1$ so this is a valid action in game 2 . In game 2 the experimenter must select a density on $\phi_{2}=A_{2}^{-1} B \theta=k^{1 / p} F \phi_{1}$. Let $a_{2}\left(\phi_{2}\right)=a_{1}\left(k^{-1 / p} F \phi_{2}\right) / k$. Now consider the actions $\left(\tau, A_{2}, y, a_{2}\right)$ in game 2. The expected reward to the experimenter is then $r_{2}^{\prime}=-\mathbb{E}_{\theta \sim \pi(\theta \mid y ; \tau)}\left[\mathcal{S}\left(a_{2}, k^{1 / p} F A_{1}^{-1} \theta\right)\right]$. From the definition of Hyvärinen score, $r_{2}^{\prime}=k^{-2 / p} r_{1}$. (As Hyvärinen score is preserved under reparameterisation by axis-aligned reflections.)

\section{Part 2: Relation between subgame perfect equilibria}

Consider a SPE of game 1 with decision rules $\tau, A(\tau), a(\tau, A, y)$. Define $A^{\prime}(\tau)=f(A(\tau))$ and $a^{\prime}(\tau, A, y)=g(a(\tau, A, y))$. Then $\tau, A^{\prime}(\tau), a^{\prime}(\tau, A, y)$ is a SPE of game 2. This follows since any counterexample could be transformed to a counterexample for the original SPE in game 1. Similarly a SPE of game 2 can be transformed to a SPE of game 1 using the inverse mappings. This suffices to prove the main result.

\section{C.5 Proof of Result 5}

\section{Part 1: Objective function for $\tau, A$}

Initially the proof follows that of Results 1 and 2. First consider the choice of $a(\tau, A, y)$ in a SPE. This must maximise the expected reward to the experimenter, which is, using $\mathrm{A} 1,-\mathbb{E}_{\phi \sim \pi_{\Phi}(\phi \mid y ; \tau)}[\mathcal{S}(a, \phi)]$. Since $\mathcal{S}$ is a strictly proper scoring rule, the optimal choice of $a$ is the posterior $\pi_{\Phi}(\phi \mid y ; \tau)$. Hence the optimal $a(\tau, A, y)$ outputs $\pi_{\Phi}(\phi \mid y ; \tau)$, and the resulting expected reward to the experimenter is the negative posterior entropy

$$
\mathbb{E}_{\phi \sim \pi_{\Phi}(\phi \mid y ; \tau)}\left[\mathcal{R}\left(\pi_{\Phi}(\phi \mid y ; \tau), \phi\right)\right]=-\mathcal{H}\left[\pi_{\Phi}(\phi \mid y ; \tau)\right]
$$

The expected reward to the experimenter for this $a$ decision rule under random $y$ is

$$
\mathbb{E}_{\phi, y \sim \pi_{\Phi}(\phi, y ; \tau)}\left[-\mathcal{H}\left[\pi_{\Phi}(\phi \mid y ; \tau)\right]\right]=\mathbb{E}_{\phi, y \sim \pi_{\Phi}(\phi, y ; \tau)}\left[\mathcal{D}\left[\pi_{\Phi}(\phi \mid y ; \tau), \pi_{\Phi}(\phi)\right]-\mathcal{H}\left[\pi_{\Phi}(\phi)\right]\right]
$$


(Noting that A5 implies that the terms above are finite and allows manipulation of expectations using Fubini's theorem.) Define $\mathcal{K}(\tau, A)$ to be the negative of the first term on the right hand side of (2). Then

$$
\begin{aligned}
\mathcal{K}(\tau, A) & =-\mathbb{E}_{\phi, y \sim \pi_{\Phi}(\phi, y ; \tau)}\left[\mathcal{D}\left[\pi_{\Phi}(\phi \mid y ; \tau), \pi_{\Phi}(\phi)\right]\right] & & \\
& =-\mathbb{E}_{\phi, y \sim \pi_{\Phi}(\phi, y ; \tau)}\left[\left\|\nabla_{\theta} \log \pi_{\Phi}(\phi \mid y ; \tau)-\nabla_{\theta} \log \pi_{\Phi}(\phi)\right\|^{2}\right] & & \text { using Table 1 } \\
& =-\mathbb{E}_{\phi \sim \pi_{\Phi}(\phi), y \sim f_{\Phi}(y \mid \phi ; \tau)}\left[\left\|\nabla_{\theta} \log f(y \mid \phi ; \tau)\right\|^{2}\right] & & \text { using (1) } \\
& =-\mathbb{E}_{\phi \sim \pi_{\Phi}(\phi)} \operatorname{tr}\left[\mathcal{I}_{\phi}(\phi ; \tau)\right] & & \text { using (4). }
\end{aligned}
$$

Note that the second line requires assumption A5. Since $\phi=A^{-1} \theta$, applying (6) gives

$$
\mathcal{K}(\tau, A)=-\mathbb{E}_{\theta \sim \pi(\theta)} \operatorname{tr}\left[A^{T} \mathcal{I}(\theta ; \tau) A\right]=-\operatorname{tr}\left[A^{T} \overline{\mathcal{I}}(\tau) A\right] .
$$

Here and in the remainder of the proof, $\pi$ and $\mathcal{I}$ without subscripts represent densities and FIM with respect to $\theta$.

Now consider the second term in $(2), \mathcal{H}\left[\pi_{\Phi}(\phi)\right]$. From Table 1 and assumption A5

$$
\mathcal{H}\left[\pi_{\Phi}(\phi)\right]=-\mathbb{E}_{\theta \sim \pi(\theta)}\left[\left\|\nabla_{\phi} \log \pi_{\Phi}(\phi)\right\|^{2}\right],
$$

where $\phi=A^{-1} \theta$. Using the change of variables formula

$$
\begin{array}{rlr}
\log \pi_{\Phi}(\phi) & =\log \pi_{\Theta}(A \phi)+\log |\operatorname{det} A| & \\
\Rightarrow \nabla_{\phi} \log \pi_{\Phi}(\phi) & =\nabla_{\theta} \log \pi_{\Theta}(\theta)|\operatorname{det} A| & \\
& =\nabla_{\theta} \log \pi_{\Theta}(\theta) & \text { since } \operatorname{det} A=1 .
\end{array}
$$

Thus $\mathcal{H}\left[\pi_{\Phi}(\phi)\right]$ equals $\mathcal{H}\left[\pi_{\Theta}(\theta)\right]$ regardless of $A$.

So under the optimal decision rule $a(\tau, A, y)$, the expected reward to the experimenter is $-\mathcal{K}(\tau, A)$, up to an additive constant. Thus the remainder of the nested optimisation problem to be solved to identify SPEs is, as claimed,

$$
\min _{\tau} \max _{A} \mathcal{K}(\tau, A)
$$

\section{Part 2: Lower bound on $\operatorname{det} \overline{\mathcal{I}}\left(\tau^{*}\right)$}

Here we prove that in a SPE, $\operatorname{det} \overline{\mathcal{I}}(\tau)>0$. This will be needed in part 4 . To show this result, we consider $\tau^{*}, A^{*}$ satisfying (4) such that $\operatorname{det} \overline{\mathcal{I}}\left(\tau^{*}\right)=0$, and derive a contradiction.

Firstly, consider the case $\mathcal{K}\left(\tau^{*}, A^{*}\right)=0$. By A4 there exists some $\tilde{\tau}$ such that $\operatorname{det} \overline{\mathcal{I}}(\tilde{\tau})>0$. Then

$$
\operatorname{det}\left[A^{* T} \overline{\mathcal{I}}(\tilde{\tau}) A^{*}\right]=\operatorname{det} \overline{\mathcal{I}}(\tilde{\tau})>0
$$

So all the eigenvalues of $A^{* T} \overline{\mathcal{I}}(\tilde{\tau}) A^{*}$ are positive. Hence

$$
\mathcal{K}\left(\tilde{\tau}, A^{*}\right)=-\operatorname{tr}\left[A^{* T} \overline{\mathcal{I}}(\tilde{\tau}) A^{*}\right]<0=\mathcal{K}\left(\tau^{*}, A^{*}\right),
$$




\section{Bayesian experimental design without posterior calculations: supplement}

which contradicts $\tau^{*}, A^{*}$ satisfying (4).

Secondly, consider the case $\mathcal{K}\left(\tau^{*}, A^{*}\right)=r<0$. Express $\overline{\mathcal{I}}\left(\tau^{*}\right)$ as an eigendecomposition $P \Lambda P^{T}$. Let $z$ be the number of zero eigenvalues (so there are $p-z$ non-zero eigenvalues). We must have $z>0$ since $\operatorname{det} \overline{\mathcal{I}}\left(\tau^{*}\right)=0$. Let $\tilde{\Lambda}(\lambda)$ be matrix resulting from replacing the zero eigenvalues in $\Lambda$ with $\lambda^{1 / z}$ and the non-zero eigenvalues with $\lambda^{-1 /(p-z)}$. Define $\tilde{A}(\lambda)=P \tilde{\Lambda}(\lambda) P^{T}$. Then $\operatorname{det} \tilde{A}(\lambda)=1$ so it is a valid action. Also $\lim _{\lambda \rightarrow \infty} \operatorname{tr}\left[\tilde{A}(\lambda)^{T} \overline{\mathcal{I}}\left(\tau^{*}\right) \tilde{A}(\lambda)\right]=0$, so for $\lambda$ sufficiently large

$$
\mathcal{K}\left(\tau^{*}, \tilde{A}(\lambda)\right)=-\operatorname{tr}\left[\tilde{A}(\lambda)^{T} \overline{\mathcal{I}}\left(\tau^{*}\right) \tilde{A}(\lambda)\right]>r=\mathcal{K}\left(\tau^{*}, A^{*}\right),
$$

which contradicts $\tau^{*}, A^{*}$ satisfying (4).

\section{Part 3: Optimal $\mathcal{K}$ given $\tau$}

Fix some $\tau$ such that $\operatorname{det} \overline{\mathcal{I}}(\tau)>0$. This part shows that $\max _{A} \mathcal{K}(\tau, A)=-p[\operatorname{det} \overline{\mathcal{I}}(\tau)]^{1 / p}$.

From $(3), \mathcal{K}(\tau, A)=-\operatorname{tr} B$ where $B=A^{T} \overline{\mathcal{I}}(\tau) A$. Let $\lambda_{1}, \ldots, \lambda_{p}$ be the eigenvalues of $B$. These are all positive: they are non-negative since $B$ is positive definite, and non-zero since $\prod_{i=1}^{p} \lambda_{i}=\operatorname{det} \overline{\mathcal{I}}(\tau)>0$. Consider minimising $\operatorname{tr} B$ subject to $\operatorname{det} A=1$, which implies $\operatorname{det} B=\operatorname{det} \overline{\mathcal{I}}(\tau)$. Equivalently we must minimise $\sum_{i=1}^{p} \lambda_{i}$ subject to $\prod_{i=1}^{p} \lambda_{i}=\operatorname{det} \overline{\mathcal{I}}(\tau)$. It is straightforward that the solution is $\lambda_{i}=[\operatorname{det} \mathcal{I}(\tau)]^{1 / p}$ for all $i$. This can be realised by taking $A$ as a Cholesky factor of $[\operatorname{det} \overline{\mathcal{I}}(\tau)]^{1 / p} \overline{\mathcal{I}}(\tau)^{-1}$. The claimed result follows from this.

\section{Part 4: Conclusion}

We will partition the possible $\tau$ values into two types. In both cases we will show that the set of those which appear in SPEs equals the set of those which attain the maximum possible $\operatorname{det} \overline{\mathcal{I}}(\tau)$ value.

First consider $\tau$ such that $\operatorname{det} \overline{\mathcal{I}}(\tau)=0$. Such a $\tau$ does not appear in a SPE (by part 2 ) and does not maximise $\operatorname{det} \overline{\mathcal{I}}(\tau)$ (from A4). Hence the two sets are empty for this case.

Next consider the case of $\tau$ such that $\operatorname{det} \overline{\mathcal{I}}(\tau)>0$. Part 1 shows that SPE actions for $\tau, A$ are solutions to $\min _{\tau} \max _{A} \mathcal{K}(\tau, A)$. Part 3 shows that, for this case, $\max _{A} \mathcal{K}(\tau, A)=$ $-p[\operatorname{det} \overline{\mathcal{I}}(\tau)]^{1 / p}$. Thus for this case, the set of designs which appear in SPEs equals those maximising $\operatorname{det} \overline{\mathcal{I}}(\tau)$, as required.

\section{C.6 Proof of Result 6}

From (13) we have

$$
\nabla_{z} \mathcal{K}(\tau, A)=-\nabla_{z} \mathbb{E}_{\theta \sim \pi(\theta)} \operatorname{tr}\left[A^{T} \mathcal{I}(\theta ; \tau) A\right] .
$$

The regularity conditions allow interchange of differentiation and expectation (see supplementary Section F) so

$$
\nabla_{z} \mathcal{K}(\tau, A)=-\mathbb{E}_{\theta \sim \pi(\theta)} \nabla_{z} \operatorname{tr}\left[A^{T} \mathcal{I}(\theta ; \tau) A\right]
$$


An unbiased Monte Carlo estimate of this is

$$
-\frac{1}{K} \sum_{k=1}^{K} \nabla_{z} \operatorname{tr}\left[A^{T} \mathcal{I}\left(\theta^{(k)} ; \tau\right) A=-\nabla_{z} \operatorname{tr}\left[A^{T}\left\{\frac{1}{K} \sum_{k=1}^{K} \mathcal{I}\left(\theta^{(k)} ; \tau\right)\right\} A\right],\right.
$$

as required.

\section{Restricting $\operatorname{det} A$}

This section justifies introducing assumption A3 in the game theoretic setting, which enforces $\operatorname{det} A=1$. First recall that we required $A$ to be invertible. For non-invertible $A, \phi=A^{-1} \theta$ would not be defined, and this is essential in the description of the game theoretic framework. Hence we must have $\operatorname{det} A \neq 0$.

Some further restriction on $A$ is necessary for a SPE to exist, at least for the scoring rules we consider. First consider the logarithmic score case. From (1) we have

$$
\log \pi_{\Phi}(\phi \mid y ; \tau)=\log \pi_{\Theta}(\theta \mid y ; \tau)+\log |\operatorname{det} A|,
$$

Without a further restriction on $A$, the critic can always improve their expected reward, $-\mathbb{E}_{\phi \sim \pi_{\Phi}(\phi \mid y ; \tau)}\left[\log \pi_{\Phi}(\phi \mid y ; \tau)\right]$, by replacing $A$ with $\lambda A$ for $\lambda<1$. (This makes the posterior for $\phi$ flatter, benefiting the critic.)

Next consider the Hyvärinen score case, and the critic's expected reward in a SPE after the experimenter plays $\tau$. From Section C.5 (part 1) this is

$$
\mathcal{K}(\tau, A)=-\mathbb{E}_{\theta \sim \pi(\theta)} \operatorname{tr}\left[A^{T} \mathcal{I}(\theta ; \tau) A\right] .
$$

Again, without a further restriction on $A$, the critic can always improve their expected reward by replacing $A$ with $\lambda A$ for $\lambda<1$. (This assumes that the expected reward is not already zero, which is a consequence of assumption A4.)

The above suggests that an additional restriction on $A$ is needed beyond $\operatorname{det} A \neq 0$. Assumption A3, $\operatorname{det} A=1$, is a natural restriction to use since $\operatorname{det} A$ appears in (5).

\section{E Point exchange algorithms}

As discussed in Section 5.4 we often wish to find a design made up of multiple design points from some region, and the optimal design is a small number of clusters of repeated observations. Gradient based optimisation can usually locate the optimal cluster locations well (i.e. determine where the clusters should be located), and here we present a simple point exchange algorithm (Atkinson et al., 2007; Overstall and Woods, 2017) to find approximately optimal cluster sizes (i.e. determine how many design points should be at each location).

The method seeks to optimise one of the diagnostics introduced in Section 5.4,

$$
\hat{\mathcal{J}}_{\mathrm{FIG}}(\tau)=\operatorname{tr}\left\{\frac{1}{J} \sum_{j=1}^{J} \mathcal{I}\left(\tilde{\theta}^{(j)} ; \tau\right)\right\}
$$




\section{Bayesian experimental design without posterior calculations: supplement}

$$
\text { or } \quad \hat{\mathcal{J}}_{\mathrm{ADV}}(\tau)=\operatorname{det}\left\{\frac{1}{J} \sum_{j=1}^{J} \mathcal{I}\left(\tilde{\theta}^{(j)} ; \tau\right)\right\} .
$$

These are Monte Carlo estimates of $\mathcal{J}_{\text {FIG }}(\tau)$ from (12) and $\mathcal{J}_{\text {ADV }}(\tau)$ from $(14)$. The $\tilde{\theta}^{(j)}$ terms are independent samples from the prior, with the same samples being reused in all evaluations of $\hat{\mathcal{J}}_{\mathrm{FIG}}$ or $\hat{\mathcal{J}}_{\mathrm{ADV}}$. Our implementation uses $J=1000$. We then seek to maximise the deterministic function $\hat{\mathcal{J}}_{\mathrm{FIG}}(\tau)$ or $\hat{\mathcal{J}}_{\mathrm{ADV}}(\tau)$.

We perform several iterations of greedy optimisation. The design $\tau^{(0)}$ is initialised as the output of gradient based optimisation. Iteration $i$ considers every possible candidate design formed from $\tau^{(i-1)}$ by replacing one design point with another. The candidate design optimising $\hat{\mathcal{J}}_{\text {FIG }}$ or $\hat{\mathcal{J}}_{\text {ADV }}$ is used as $\tau^{(i)}$. The algorithm terminates if $\tau^{(i)}=\tau^{(i-1)}$ or if a maximum number of iterations is exceeded.

Note that this is a simple approximate optimisation method, and there is scope for more sophisticated algorithms to be developed for use here.

\section{F Regularity conditions}

Several of our derivations, such as Result 6 , require weak regularity conditions on $g(\theta, \tau)$ to allow interchange of differentiation and expectation i.e.

$$
\nabla_{\tau} \mathbb{E}_{\theta \sim \pi(\theta)}[g(\theta, \tau)]=\mathbb{E}_{\theta \sim \pi(\theta)}\left[\nabla_{\tau} g(\theta, \tau)\right] .
$$

Sufficient regularity conditions are that for all $\tau$,

1. $\mathbb{E}_{\theta \sim \pi(\theta)}[|g(\theta, \tau)|]$ is finite

2. $g(\theta, \tau)$ is differentiable in each component of $\tau$

3. $\mathbb{E}_{\theta \sim \pi(\theta)}\left[\left|\nabla_{\theta} g(\theta, \tau)\right|\right]$ is finite

where absolute value $|\cdot|$ acts elementwise. The required equality then follows using Fubini's theorem.

\section{G Intractable Fisher information}

This section considers how to apply our approach when the FIM is not available in a closed form that can easily be evaluated.

First we consider two cases where extending our approach is relatively straightforward, and give an algorithm which does so. Section G.1 looks at the case where the score function is available, and Section G.2 at the case where the likelihood is available. In the latter we also discuss scope for future work to improve the efficiency of the algorithm's implementation in code.

Secondly we discuss two more complex cases which we plan to consider in future work. At the request of a reviewer, we sketch methods to do so, to show that this is feasible. Section G.3 looks at the case where there are latent variables, such as nuisance parameters, and Section G.4 at the case where the likelihood is intractable. 
Recap Recall that our ADV approach is to find minimax solutions of

$$
\mathcal{K}(\tau, A)=-\mathbb{E}_{\theta \sim \pi(\theta)} \operatorname{tr}\left[A^{T} \mathcal{I}(\theta ; \tau) A\right],
$$

using gradient descent ascent. As in the main paper we take $A=A(\eta)$, following (15). GDA requires unbiased estimates of $\nabla_{z} \mathcal{K}(\tau, A)$ for $z \in\{\tau, \eta\}$.

\section{G.1 Estimation using the score function}

First we consider the case where the score function $u(y, \theta ; \tau)=\nabla_{\theta} \log f(y \mid \theta ; \tau)$ can be evaluated. We show that it is possible to produce unbiased estimates of the required gradients, provided assumption A6, stated below, is met. This allows us to perform optimisation using GDA, as described below in Algorithm 1.

Estimation of objective Recall that (4) defines

$$
\mathcal{I}_{\theta}(\theta ; \tau)=\mathbb{E}_{y \sim f(y \mid \theta ; \tau)}\left[u(y, \theta ; \tau) u(y, \theta ; \tau)^{T}\right] .
$$

Substituting this into (6) gives

$$
\begin{aligned}
\mathcal{K}(\tau, A) & =-\mathbb{E}_{\theta \sim \pi(\theta), y \sim f(y \mid \theta ; \tau)} \operatorname{tr}\left[A^{T} u(y, \theta ; \tau) u(y, \theta ; \tau)^{T} A\right] \\
& =-\mathbb{E}_{\theta \sim \pi(\theta), y \sim f(y \mid \theta ; \tau)}\left[u(y, \theta ; \tau)^{T} A A^{T} u(y, \theta ; \tau)\right] \\
& =-\mathbb{E}_{\theta \sim \pi(\theta), y \sim f(y \mid \theta ; \tau)}\left[\left\|A^{T} u(y, \theta ; \tau)\right\|^{2}\right],
\end{aligned}
$$

which can easily be estimated unbiasedly by Monte Carlo.

Estimation of gradients Getting unbiased estimators of $\nabla_{z} \mathcal{K}(\tau, A)$ is more complicated. This is because on taking the gradient of (8), it is generally not possible to exchange the order of gradient and expectation operators, as the distribution for $y$ depends on $\tau$. Therefore we use the pathwise derivative approach (see Mohamed et al., 2020 for a review) based on the following assumption:

A6 The observations $y$ can be generated as a transformation $y(\epsilon, \theta, \tau)$ of a random variable $\epsilon$ with fixed density $p(\epsilon)$.

This gives

$$
\mathcal{K}(\tau, A)=-\mathbb{E}_{\theta \sim \pi(\theta), \epsilon \sim p(\epsilon)}\left[\left\|A^{T} u(y(\epsilon, \theta, \tau), \theta ; \tau)\right\|^{2}\right] .
$$

Now it is possible to exchange expectation and differentiation, assuming appropriate regularity conditions (see supplementary Section F),

$$
\nabla_{z} \mathcal{K}(\tau, A)=-\mathbb{E}_{\theta \sim \pi(\theta), \epsilon \sim p(\epsilon)}\left[\nabla_{z}\left\|A^{T} u(y(\epsilon, \theta, \tau), \theta ; \tau)\right\|^{2}\right] .
$$

Hence an unbiased Monte Carlo estimate of $\mathcal{K}(\tau, A)$ is

$$
\breve{\mathcal{K}}(\tau, A)=-\frac{1}{K} \sum_{k=1}^{K}\left[\left\|A^{T} u\left(y\left(\epsilon^{(k)}, \theta^{(k)}, \tau\right), \theta^{(k)} ; \tau\right)\right\|^{2}\right],
$$




\section{Bayesian experimental design without posterior calculations: supplement}

where $\left(\theta^{(k)}, \epsilon^{(k)}\right)$ are independent draws from $\pi(\theta) p(\epsilon)$. Also, it follows from the above that $\nabla_{z} \breve{\mathcal{K}}(\tau, A)$ is an unbiased estimate of $\nabla_{z} \mathcal{K}(\tau, A)$ for $z \in\{\tau, \eta\}$.

In some cases A6 does not hold, as $y$ cannot be represented as a suitable transformation of $\epsilon$. This includes the case of discrete $y$ and other common distributions such as Beta and Gamma. Various techniques are available to deal with these cases such as taking a continuous approximation to discrete variables (Maddison et al., 2017) or using implicit differentiation (Figurnov et al., 2018; Jankowiak and Obermeyer, 2018).

Algorithm Algorithm 1 presents our approach when the score function can be evaluated, based on the use of pathwise derivative gradient estimates.

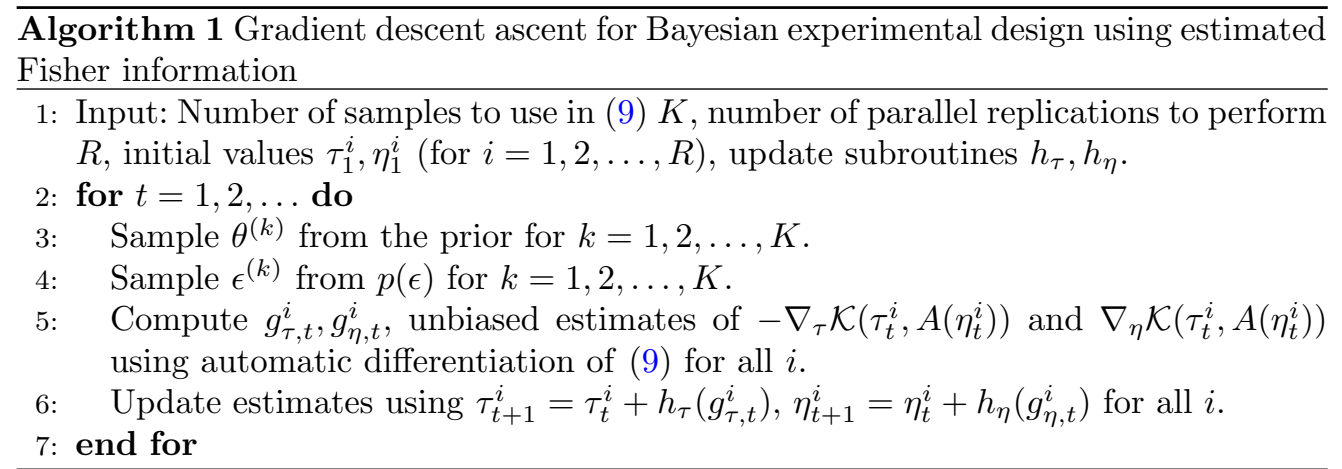

\section{G.2 Estimation using the likelihood}

Here we consider the case where the likelihood can be evaluated easily but the score function cannot. In this case whenever the score function is required it can simply be calculated by automatic differentiation of the log likelihood, and we can use Algorithm 1. Section 7.5 has an example using this approach, with further details in supplementary Section J.

Our PyTorch code for this case is restricted to $R=K=1$ i.e. number of parallel replications and Monte Carlo sample size must both equal one. Otherwise an iteration of the algorithm requires calculating likelihood gradients for several $(\theta, y)$ combinations, then manipulating these, and finally calculating gradients again: it is difficult to perform such a calculation efficiently in PyTorch. In future work it would be interesting to implement this method in other frameworks which provide more flexibility in the use of automatic differentiation, such as Jax (Bradbury et al., 2018).

\section{G.3 Latent variables case}

Next we discuss a case where the likelihood cannot be evaluated as the observations depend on latent variables $\psi$. The latent variables could represent: 
- Nuisance parameters that we do not wish to learn from our experiment, but which affect the distribution of $y$ nonetheless.

- Unobserved states in a time series model.

- Model parameters when we are interested only in model choice.

Below we sketch an approach to experimental design in the presence of latent variables. This illustrates the difficulty of this case but that progress is feasible. We plan to investigate this further in future work.

Estimation of score The observations now depend on latent variables $\psi$, so their density is $f(y \mid \theta, \psi ; \tau)$. Let the conditional prior density of $\psi$ be $\pi(\psi \mid \theta)$. We would like to work with the marginalised likelihood

$$
f(y \mid \theta ; \tau)=\int f(y \mid \theta, \psi ; \tau) \pi(\psi \mid \theta) d \psi,
$$

and calculate the corresponding marginalised score function $\nabla_{\tau} \log f(y \mid \theta ; \tau)$ and the resulting Fisher information matrix. Unfortunately the marginalised likelihood involves an intractable integral, which typically cannot be evaluated.

However an unbiased estimate of the marginalised score function exists, using Fisher's identity (Cappé et al., 2006; Poyiadjis et al., 2011)

$$
u(y, \theta ; \tau)=\nabla_{\theta} \log f(y \mid \theta ; \tau)=\mathbb{E}_{\psi \sim \pi(\psi \mid \theta, y ; \tau)}\left[\nabla_{\theta} \log f(y, \psi \mid \theta ; \tau)\right] .
$$

where $\pi(\psi \mid \theta, y ; \tau)$ is a posterior for the latent variables conditional on $\theta$.

Difficulties The two difficulties in implementing the ADV approach with latent variables are:

1. Inference for $\pi(\psi \mid \theta, y ; \tau)$.

2. Getting unbiased estimates of $\nabla_{\tau} u(y, \theta ; \tau)$.

The latter is required in estimating $\nabla_{\tau} \mathcal{K}(\tau, A)$, and it is difficult because on taking the gradient of (10), it is not possible to exchange the order of gradient and expectation operators, as the posterior distribution for $\psi$ depends on $\tau$.

Below we sketch an approach which deals with both difficulties.

Estimation of $\psi$ posterior For now, suppose $\tau$ is fixed. We would like to have access to $\pi(\psi \mid \theta, y ; \tau)$ for any choices of $\theta$ and $y$. An approximate approach is to use a version of neural density estimation. The goal is to find a function $g(\varepsilon, \theta, y)$ which given $\varepsilon \sim N(0, I)$ outputs samples from a close estimate of $\pi(\psi \mid \theta, y ; \tau)$. The function $g$ can be a neural network of suitable form, such as a normalising flow (Papamakarios et al., 2021). The neural network's tuning parameters can be trained by using SGD to maximise the likelihood based on samples of $(\theta, \psi, y)$ from the prior and model (Papamakarios et al., 2021). See Foster et al. (2019, 2020); Kleinegesse and Gutmann (2020) for approaches to Bayesian experimental design under the SIG objective which make use of neural density estimation or closely related methods. 


\section{Bayesian experimental design without posterior calculations: supplement}

Estimation of objective gradients Suppose one has access to a function $g(\varepsilon, \theta, y)$ which given $\varepsilon \sim N(0, I)$ produces samples from $\pi\left(\psi \mid \theta, y ; \tau^{*}\right)$ where $\tau^{*}$ is the optimal design. Fisher's identity (10) then becomes

$$
u(y, \theta ; \tau)=\mathbb{E}_{\varepsilon \sim N(0, I)}\left[\nabla_{\theta} \log f(y, \psi \mid \theta ; \tau)\right] .
$$

where $\psi$ is a shorthand for $^{1} g(\varepsilon, \theta, y)$. Similarly, we will assume A6 and write $y$ as shorthand for $y(\epsilon, \theta, \tau)$. Substituting (11) into (7) gives

$$
\begin{aligned}
\mathcal{K}(\tau, A) & =-\mathbb{E}_{\theta \sim \pi(\theta), \epsilon \sim p(\epsilon), \varepsilon_{1} \sim N(0, I), \varepsilon_{2} \sim N(0, I)}\left[\log f\left(y, \psi_{1} \mid \theta ; \tau\right)^{T} A A^{T} \log f\left(y, \psi_{2} \mid \theta ; \tau\right)\right], \\
\nabla_{z} \mathcal{K}(\tau, A) & =-\mathbb{E}_{\theta \sim \pi(\theta), \epsilon \sim p(\epsilon), \varepsilon_{1} \sim N(0, I), \varepsilon_{2} \sim N(0, I)}\left[\nabla_{z}\left\{\log f\left(y, \psi_{1} \mid \theta ; \tau\right)^{T} A A^{T} \log f\left(y, \psi_{2} \mid \theta ; \tau\right)\right\}\right] .
\end{aligned}
$$

Here that $\varepsilon_{1}$ and $\varepsilon_{2}$ are independent $N(0, I)$ random vectors, and $\psi_{1}, \psi_{2}$ are shorthand for $g\left(\varepsilon_{1}, \theta, y\right)$ and $g\left(\varepsilon_{2}, \theta, y\right)$.

The final expression above allows Monte Carlo estimation of the required gradients of the objective $\mathcal{K}(\tau, A)$.

Optimisation algorithm We can now sketch an algorithm for optimal design, which is to iterate the following steps:

1. Sample $\theta, \psi, \epsilon$ given the current $\tau$.

2. Update $g$ by a step of SGD based on training data $(\theta, \psi, y(\epsilon, \theta, \tau))$.

3. Sample $\varepsilon_{1}, \varepsilon_{2}$.

4. Produce unbiased estimates of $\nabla_{\tau} \mathcal{K}$ and $\nabla_{\eta} \mathcal{K}$.

5. Update $\tau$ and $\eta$ by SGD steps.

Upon convergence of $\tau$ to a limit point $\tau^{*}, g$ will approximate the corresponding posterior for $\psi$. Hence $\tau^{*}$ will approximate an optimal design.

\section{G.4 Intractable likelihood}

Finally, consider the case where it is only possible to sample $y$ given $\theta, \tau$. In this case we do not have access to an unbiased estimator of the score function. However it is possible to train an estimate of the likelihood $\hat{f}(y \mid \theta)$ using neural density estimation methods. This estimate can be used to define a score estimate $\hat{u}(y, \theta)=\nabla_{\theta} \log \hat{f}(y \mid \theta)$, allowing the approach of the previous subsection to be used. The difference is that now $u$ is estimated purely from simulations, while the previous section also made use of the full likelihood via (10).

\section{H Poisson example derivations}

Here we derive the results stated in Section 6 on the Poisson example. Recall that the design is $\tau \in[0,1]$, and the observations are $y_{1} \sim \operatorname{Poisson}\left(\tau \theta_{1} \omega_{1}\right), y_{2} \sim \operatorname{Poisson}((1-$ $\left.\tau) \theta_{2} \omega_{2}\right)$, and these are independent. We assume $\omega_{1}>\omega_{2}>0$ and that $\theta_{1}, \theta_{2}$ have independent $\operatorname{Gamma}(2,1)$ priors.

\footnotetext{
${ }^{1}$ Note that $\epsilon$ and $\varepsilon$ represent different random vectors.
} 
Posterior distribution Using standard conjugacy relationships we have independent posterior distributions $\theta_{1}\left|y_{1} \sim \operatorname{Gamma}\left(2+y_{1}, 1+\tau \omega_{1}\right), \theta_{2}\right| y_{2} \sim \operatorname{Gamma}\left(2+y_{2}, 1+\right.$ $\left.(1-\tau) \omega_{2}\right)$. Note that if $\tau=0$ then $y_{1}$ is a point mass at zero, and so the $\theta_{1}$ posterior equals its prior. Similarly if $\tau=1$ then the $\theta_{2}$ posterior equals its prior.

Expected Fisher information matrix For independent observations depending on different parameters, the FIM is diagonal with entries given by the scalar Fisher informations of the individual parameters (this is a straightforward consequence of the definition (4)). Now consider $y \sim \operatorname{Poisson}(\varphi)$. The Fisher information with respect to $\varphi$ is $1 / \varphi$. Suppose $\varphi=k \vartheta$. Using (6), the Fisher information with respect to $\vartheta$ is $k^{2} / \varphi=k / \vartheta$.

Hence the FIM for this example is:

$$
\mathcal{I}_{\theta}(\theta)=\left(\begin{array}{cc}
\tau \omega_{1} / \theta_{1} & 0 \\
0 & (1-\tau) \omega_{2} / \theta_{2}
\end{array}\right)
$$

Note that $1 / \theta_{i}$ has an inverse gamma $(2,1)$ distribution which has expectation 1 . Thus

$$
\overline{\mathcal{I}}_{\theta}(\tau)=\left(\begin{array}{cc}
\tau \omega_{1} & 0 \\
0 & (1-\tau) \omega_{2}
\end{array}\right) .
$$

ADV design We have $\operatorname{det}\left[\overline{\mathcal{I}}_{\theta}(\theta)\right]=\tau(1-\tau) \omega_{1} \omega_{2}$ which is maximised by the design $\tau=1 / 2$. Furthermore we have already shown in Result 4 that this design is invariant to linear reparameterisation.

FIG design We have $\operatorname{tr}\left[\overline{\mathcal{I}}_{\theta}(\theta)\right]=\tau \omega_{1}+(1-\tau) \omega_{2}$. Since $\omega_{1}>\omega_{2}$, this is maximised by $\tau=1$.

Next, consider a linear reparameterisation $\phi=B \theta$. Then

$$
\begin{aligned}
\overline{\mathcal{I}}_{\phi}(\phi) & =\mathbb{E}_{\theta \sim \pi(\theta)}\left[\mathcal{I}_{\phi}(\phi)\right] & & \\
& =\mathbb{E}_{\theta \sim \pi(\theta)}\left[B^{-T} \mathcal{I}_{\theta}(\theta) B^{-1}\right] & & \text { from }(6) \\
\Rightarrow \operatorname{tr} \overline{\mathcal{I}}_{\phi}(\theta) & =\operatorname{tr}\left[B^{-1} B^{-T} \overline{\mathcal{I}}_{\theta}(\theta)\right] & & \text { by linearity of expectation and } \\
& =\tau \omega_{1}^{\prime}+(1-\tau) \omega_{2}^{\prime} & & \text { cyclic property of trace }
\end{aligned}
$$

where $\left(\begin{array}{l}\omega_{1}^{\prime} \\ \omega_{2}^{\prime}\end{array}\right)$ is the elementwise product of $\operatorname{diag}\left(B^{-1} B^{-T}\right)$ and $\left(\begin{array}{l}\omega_{1} \\ \omega_{2}\end{array}\right)$. The resulting optimal design is

- $\tau=1$ if $\omega_{1}^{\prime}>\omega_{2}^{\prime}$

- $\tau=0$ if $\omega_{1}^{\prime}<\omega_{2}^{\prime}$

- $\tau \in[0,1]$ if $\omega_{1}^{\prime}=\omega_{2}^{\prime}$

and clearly $B$ can be chosen to allow all these possibilities. 


\section{Bayesian experimental design without posterior calculations: supplement}

SIG design Without loss of generality suppose $\omega_{1}=\omega_{2}=1$. This is equivalent to a suitable reparameterisation of $\theta$, and the SIG design is invariant to reparameterisation.

For any $\tau<1 / 2$, let $h=1 / 2-\tau$ and define $X_{1} \sim \operatorname{Poisson}(\tau \theta), X_{2} \sim \operatorname{Poisson}(h \theta)$, $X_{3} \sim \operatorname{Poisson}(h \theta)$. Now let $S_{i}=\sum_{j=1}^{i} X_{i}$. so that $S_{1} \sim \operatorname{Poisson}(\tau \theta), S_{2} \sim \operatorname{Poisson}(\theta / 2)$, $S_{3} \sim \operatorname{Poisson}([1-\tau] \theta)$. Note that $X_{1}, X_{2}, X_{3}$ are not independent, but are conditionally independent given $\theta$.

Define $v(\mathcal{A})$ as the expected Shannon information gain for parameter $\theta$ upon observing the set of variables $\mathcal{A}$. Using sufficiency, we get $v\left(S_{1}\right)=v\left(X_{1}\right), v\left(S_{2}\right)=$ $v\left(\left\{X_{1}, X_{2}\right\}\right)=v\left(\left\{X_{1}, X_{3}\right\}\right)$ and $v\left(S_{3}\right)=v\left(\left\{X_{1}, X_{2}, X_{3}\right\}\right)$. We prove below that

$$
v\left(\left\{X_{1}, X_{2}\right\}\right)-v\left(X_{1}\right)>v\left(\left\{X_{1}, X_{2}, X_{3}\right\}\right)-v\left(\left\{X_{1}, X_{3}\right\}\right),
$$

so that $v\left(S_{2}\right)-v\left(S_{1}\right)>v\left(S_{3}\right)-v\left(S_{2}\right)$. Rearranging gives $2 v\left(S_{2}\right)>v\left(S_{3}\right)+v\left(S_{1}\right)$.

In our experiment we have two observations $Y_{1}, Y_{2}$, which are conditionally independent given $\theta$. It follows from the definition of Shannon information gain that $v\left(\left\{Y_{1}, Y_{2}\right\}\right)=v\left(Y_{1}\right)+v\left(Y_{2}\right)$. Hence the expected Shannon information gain from the experiment is $v\left(S_{3}\right)+v\left(S_{1}\right)$ under design $\tau$ (or $1-\tau$ ), and $2 v\left(S_{2}\right)$ under design $1 / 2$. Thus we have shown that the latter is optimal.

Proof of (12) Here we adapt the proof of proposition 2 from Krause and Guestrin (2005) (on submodularity of mutual information). Define $\mathcal{X}$ to be a subset of $\left\{X_{1}, X_{2}, X_{3}\right\}$. From Bayes theorem and conditional independence we have that

$$
\begin{aligned}
\log \pi(\theta \mid \mathcal{X})-\log \pi(\theta) & =\log \operatorname{Pr}(\mathcal{X})+\log \operatorname{Pr}(\mathcal{X} \mid \theta) \\
& =\log \operatorname{Pr}(\mathcal{X})+\sum_{X \in \mathcal{X}} \log \operatorname{Pr}(X \mid \theta)
\end{aligned}
$$

Taking the expectation with respect to $\theta$ and all $X$ variables gives

$$
v(\mathcal{X})=H(\mathcal{X})+\sum_{X \in \mathcal{X}} H\left(X^{\prime}\right)
$$

where $H$ denotes Shannon entropy and the dash denotes conditioning on $\theta$. Applying this result to both sides of (12) gives

$$
\begin{aligned}
v\left(\left\{X_{1}, X_{2}\right\}\right)-v\left(X_{1}\right) & =H\left(\left\{X_{1}, X_{2}\right\}\right)-H\left(X_{1}\right)+H\left(X_{2}^{\prime}\right) \\
v\left(\left\{X_{1}, X_{2}, X_{3}\right\}\right)-v\left(\left\{X_{1}, X_{3}\right\}\right) & =H\left(\left\{X_{1}, X_{2}, X_{3}\right\}\right)-H\left(\left\{X_{1}, X_{2}\right\}\right)+H\left(X_{2}^{\prime}\right) .
\end{aligned}
$$

To prove (12) it remains to demonstrate the inequality

$$
H\left(\left\{X_{1}, X_{2}\right\}\right)-H\left(X_{1}\right)=H\left(X_{2} \mid X_{1}\right)>H\left(X_{2} \mid\left\{X_{1}, X_{3}\right\}\right)=H\left(\left\{X_{1}, X_{2}, X_{3}\right\}\right)-H\left(\left\{X_{1}, X_{2}\right\}\right) .
$$

This holds due to the "information can't hurt" principle (see e.g. Cover and Thomas, 2012, Theorem 2.6.5), with the non-independence of the $X_{i}$ variables giving strict inequality. 


\section{Further details of pharmacokinetic example}

\section{I.1 Fisher information matrix}

The pharmacokinetic model is of the form $y \sim N\left(x(\theta, \tau), \sigma^{2} I\right)$ with $\sigma$ fixed and $\operatorname{dim} x=$ $n$. Using (7),

$$
\mathcal{I}_{\theta}(\theta)=\sigma^{-2} J^{T} J
$$

where $J$ is a Jacobian matrix, with row $i$ column $j$ entry $\frac{\partial x_{i}}{\partial \theta_{j}}$. It remains to state these terms, which are

$$
\begin{aligned}
\frac{\partial}{\partial \theta_{1}} x_{i}(\theta, \tau) & =\frac{1}{\theta_{2}-\theta_{1}} x\left(\theta, \tau_{i}\right)-\frac{D \theta_{2}}{\theta_{3}\left(\theta_{2}-\theta_{1}\right)} \tau_{i} \exp \left(-\theta_{1} \tau_{i}\right), \\
\frac{\partial}{\partial \theta_{2}} x_{i}(\theta, \tau) & =\frac{\theta_{1}}{\theta_{2}\left(\theta_{1}-\theta_{2}\right)} x\left(\theta, \tau_{i}\right)+\frac{D \theta_{2}}{\theta_{3}\left(\theta_{2}-\theta_{1}\right)} \tau_{i} \exp \left(-\theta_{2} \tau_{i}\right), \\
\frac{\partial}{\partial \theta_{3}} x_{i}(\theta, \tau) & =-\frac{1}{\theta_{3}} x\left(\theta, \tau_{i}\right) .
\end{aligned}
$$

\subsection{Choice of $K$}

Section 7.2 mentions that, when performing the GDA analysis of the pharmacokinetic example, we compared several choices of $K$, the number samples to estimate $\mathcal{K}$ in (16). Figure 1 shows the results of using $K=1,10,100$ by plotting $\mathcal{J}_{\mathrm{ADV}}$ estimates, as defined in (17). It shows that convergence is slowest for $K=100$. We use $K=1$ in the main paper as it appears slightly quicker than $K=10$.

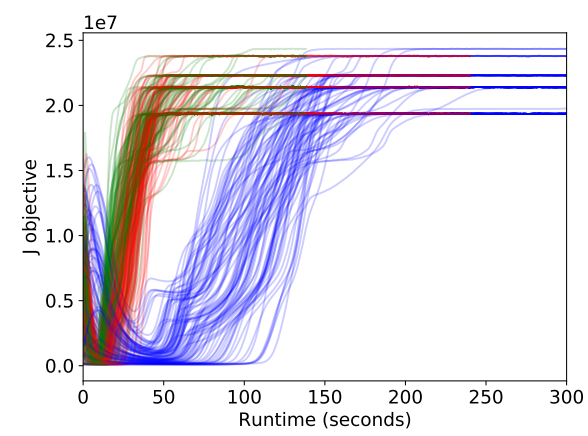

Figure 1: Trace plots of runtime versus $\mathcal{J}_{\text {ADV }}$ estimates for 100 replications of GDA on the pharmacokinetic example for $K=1$ (green), $K=10$ (red), $K=100$ (blue). The runtime shown is the total runtime for all parallel replications. 


\section{I.3 Results using Overstall and Woods (2017) approach}

Here we give some further details of our implementation of the pharmacokinetic example using the ACE method of Overstall and Woods (2017) for SIG optimisation. The analysis presented in the main paper uses the default tuning choices in Overstall and Woods (2017). As mentioned in the main text, we experimented with one tuning choice but found little improvement. The implementation uses the acebayes package (Overstall et al., 2019), and our code can be found at https://github.com/dennisprangle/ AdversarialDesignCode/R.

The ACE software offers a second phase of point exchange optimisation. Exploratory analysis showed that using this makes little change to the designs found for this example. Therefore to avoid the extra computational cost, we did not use it for our main analysis.

Figure 2 shows the designs produced for 30 runs from different initial designs. The design points are spread out over the entire $[0,24]$ interval, although not uniformly. This suggests that ACE has not completely converged, as the SIG optimisation results using approaches from Foster et al. (2020) (see next subsection) are much less spread out.

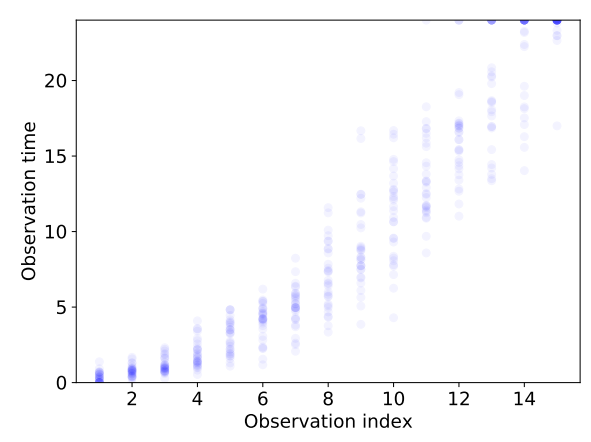

Figure 2: Designs output by 30 runs of ACE for the pharmacokinetic example. The horizontal axis shows the index of each point in the sorted design i.e. observation times are shown in increasing order from left to right.

\section{I.4 Results using Foster et al. (2020) approaches}

The main paper presents results for SIG optimisation for the pharmacokinetic example using methods from Foster et al. (2020). An adaptation of their code to reproduce our analysis is available at https://github.com/dennisprangle/pyro/tree/ sgboed-reproduce. Foster et al. (2020) propose several methods. First we discuss prior contrastive estimation (PCE), as we found it converged most quickly for this application, and is therefore the method we report in the main paper to give a lower bound on the speed for methods from this paper.

To investigate convergence of $\mathrm{PCE}$ we considered designs produced after 1 and 2 hours. Figure 3 shows these designs, for 100 replications of the optimisation algorithm, 
which were computed in parallel. The designs after 2 hours are slightly different, suggesting that the method has not yet fully converged. In the main paper we report results after 1 hour, taking this as a lower bound on the time required for convergence.

Figure 3 has 3 clusters of design points, around ${ }^{2} t=1,4,14$. The points are more spread out around these clusters than the ADV results from the main paper, but this may be due to incomplete convergence, as discussed shortly.
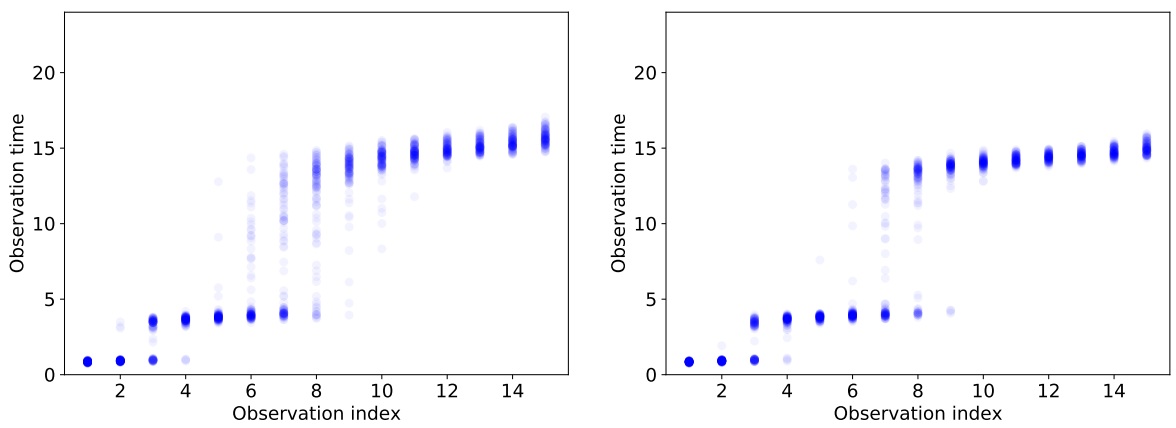

Figure 3: Designs output by 100 runs of PCE for the pharmacokinetic example after 1 hour (left) and 2 hours (right). The horizontal axis shows the index of each point in the sorted design i.e. observation times are shown in increasing order from left to right.

Foster et al. (2020) propose several alternatives to PCE, and indeed PCE is not their overall recommendation. For instance it is only guaranteed to converge to the optimal design in the limit of a large number of Monte Carlo samples being used to estimate gradients in each iteration. However for this example we found the other methods were slower or less numerically stable, as we describe now.

We also attempted to implement the Barber-Agakov (BA) and adaptive contrastive estimation $\left(\mathrm{ACE}^{3}{ }^{3}\right.$ ) methods of Foster et al. (2020). Both methods involve a neural network to map observations $y$ to an approximate posterior for $\theta, N(\mu(y), \Sigma(y))$. We used a neural network with 2 fully connected layers of 16 hidden units and ReLU activation functions. A final linear layer produces 9 outputs: 3 are used for $\mu$ and the other 6 for a Cholesky factor for $\Sigma(y)$.

We were unable to implement ACE2 as training produced NaN outputs. So we discuss only the results of BA. We ran 100 replications in parallel. The left plot in Figure 4 shows the results of one well-behaved replication. The design points converge to 3 clusters near $t=0.7,4.5,15.4$ after roughly 5,000 seconds (1.4 hours). These are similar but not identical to the cluster locations for ADV reported in the main paper.

\footnotetext{
${ }^{2}$ We report these only to the nearest integer, which is less precise than cluster locations for other methods elsewhere in the paper. This is because the design points here are more variable across replications.

${ }^{3}$ We refer to this as ACE2 to distinguish it from the ACE method of Overstall and Woods (2017) discussed elsewhere in the paper.
} 


\section{Bayesian experimental design without posterior calculations: supplement}

However many other replications did not converge so clearly to these locations, as shown by the right plot in Figure 4. Indeed, in a few replications we noticed the design remained very close to its initial values.
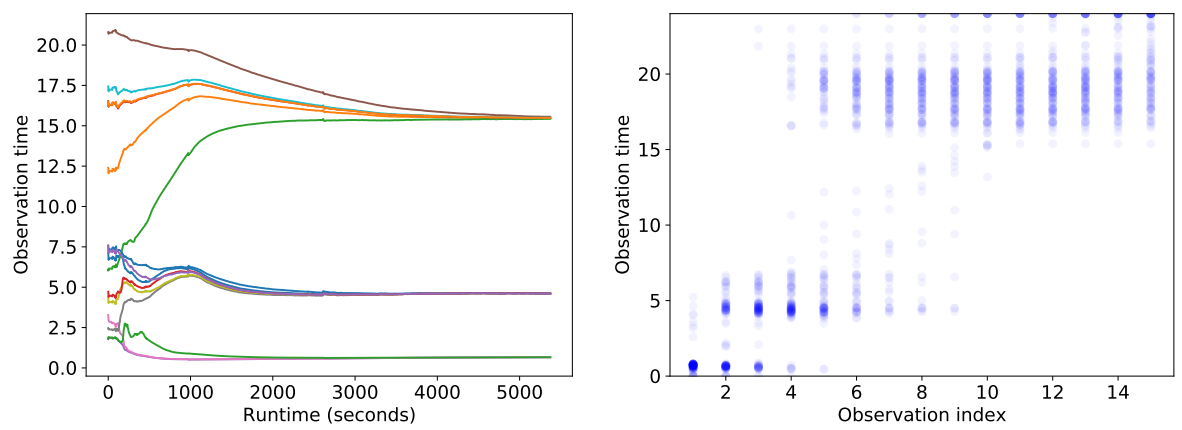

Figure 4: Left: Trace plot for a single replication of BA on the pharmacokinetic example. 100 replications were run in parallel and the $x$-axis shows the total time to run all these replications. Right: Designs output by all replications after 18 hours. The horizontal axis shows the index of each point in the sorted design i.e. observation times are shown in increasing order from left to right.

The BA runtime for the well-behaved replication is a little longer than the time used for PCE in the main paper - which we ran for 1 hour (3,600 seconds) for 100 replications in parallel - but of a similar order of magnitude. However other replications did not appear to have converged after running for several hours. As discussed by Foster et al. (2020), BA is likely to be somewhat slower than PCE as it needs to optimise a large number of neural network weights in addition to the design.

We expect that with further effort BA and ACE2 can be more successfully implemented for this example. However, we believe that the PCE results reported in the main paper capture a lower bound on the runtime for any of these methods on this example.

\section{I.5 SGD results}

Here we consider using the FIG approach rather than ADV for the pharmacokinetic example. To do so, Figure 5 shows results of optimising $\mathcal{J}_{\text {FIG }}$ using the SGD version of Algorithm 2. The top left plot shows the evolution of the design during a single replication. The design points converge to repeated observations at a single time. The top right and bottom left plots display estimated $\mathcal{K}$ and $\mathcal{J}_{\text {FIG }}$ objectives over 100 repeated runs, which show rapid convergence. The bottom right plot shows that in 100 runs, the design points always converge to a single cluster, around time 12. Point exchange was not implemented as multiple clusters were not observed. 

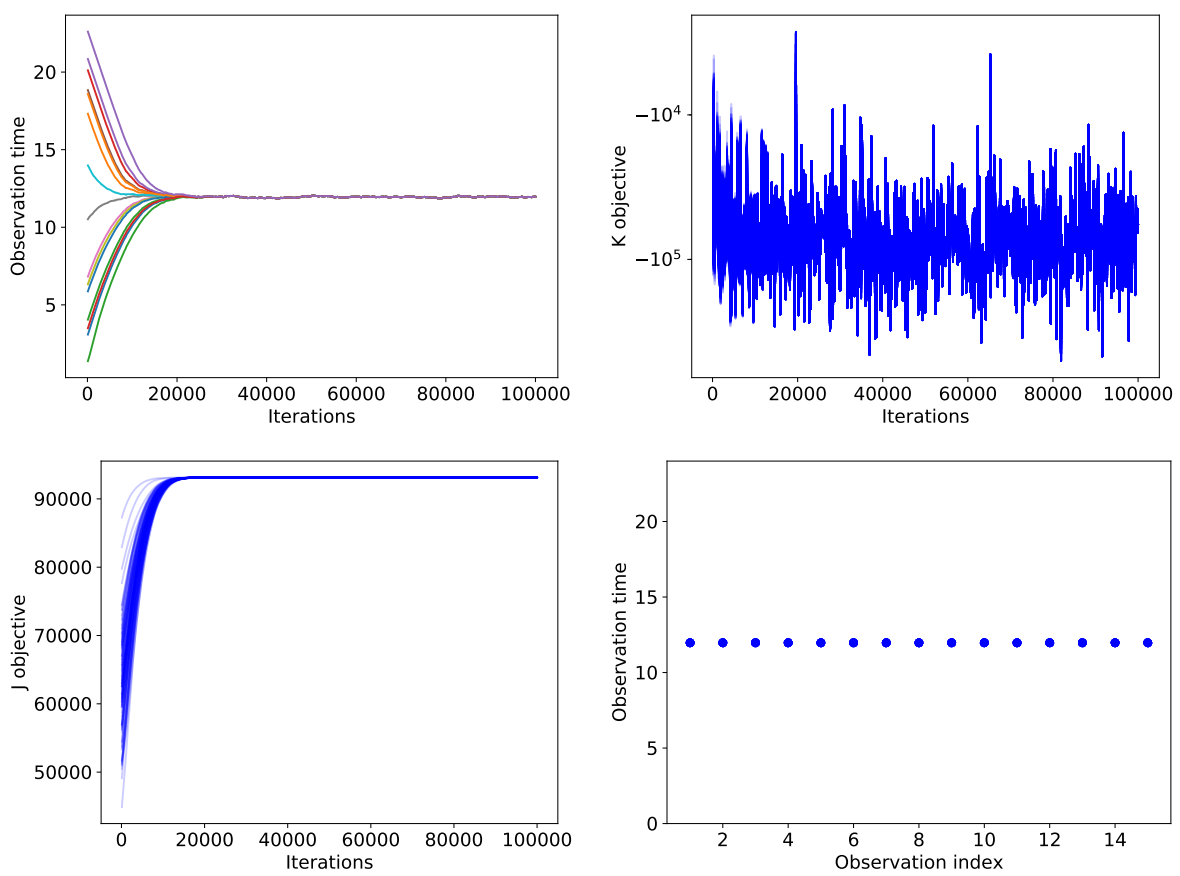

Figure 5: Summary plots for FIG on the pharmacokinetic example. Top left: designs during a single run of SGD. Top right: $\mathcal{K}$ objective for 100 runs. Bottom left: $\mathcal{J}_{\text {FIG }}$ objective for 100 runs. Bottom right: designs output from 100 SGD runs. The horizontal axis shows the index of each point in the sorted design i.e. observation times are shown in increasing order from left to right. The designs are not identical between runs but the variation is too small to see on this scale. 
20 Bayesian experimental design without posterior calculations: supplement

\section{I.6 Shannon information gain}

Figure 4 shows expected Shannon information gain values as a diagnostic for pharmacokinetic example design quality. This subsection derives the calculations used for this diagnostic.

From (8) and (9), the expectation of the Shannon information gain utility is, in general

$$
\mathcal{J}_{\text {SIG }}(\tau)=\mathbb{E}_{(\theta, y) \sim \pi(\theta, y ; \tau)}[\log f(y \mid \theta ; \tau)-\log \pi(y ; \tau)],
$$

where $f(y \mid \theta ; \tau)$ is the likelihood and $\pi(y ; \tau)$ is the evidence.

The pharmacokinetic model has the form $y=x(\theta, \tau)+\epsilon$ where $\epsilon \sim N\left(0, \sigma^{2} \mathrm{I}\right)$. Let $\varphi(\epsilon)$ be the corresponding density. Then:

$$
\mathbb{E}_{y \sim f(y \mid \theta ; \tau)}[\log f(y \mid \theta ; \tau)]=\mathbb{E}_{\epsilon}[\log \varphi(\epsilon)]=-H(\epsilon),
$$

where $H$ represents Shannon entropy. Using the standard result for the Shannon entropy of the multivariate normal (see e.g. Cover and Thomas, 2012, Theorem 8.4.1) gives

$$
H(\epsilon)=\frac{\operatorname{dim} y}{2}[1+\log (2 \pi)+2 \log \sigma]=-13.25 \quad \text { (to } 2 \text { decimal places) }
$$

since $\operatorname{dim} y=15$ and $\sigma=0.1$. Hence

$$
\mathcal{J}_{\mathrm{SIG}}(\tau)=H(\epsilon)-\mathbb{E}_{(\theta, y) \sim \pi(\theta, y ; \tau)} \log \pi(y ; \tau) .
$$

The second term can be estimated using a nested Monte Carlo approximation, to give

$$
\begin{aligned}
\hat{\mathcal{J}}_{\mathrm{SIG}}(\tau) & =H(\epsilon)-\frac{1}{B} \sum_{i=1}^{B} \log \hat{\pi}\left(y^{(i)} ; \tau\right), \\
\text { where } \quad \hat{\pi}(y ; \tau) & =\frac{1}{B^{\prime}} \sum_{j=1}^{B^{\prime}} f\left(y \mid \theta^{(j)} ; \tau\right) .
\end{aligned}
$$

Here the $y^{(i)} \mathrm{s}$ are independent samples from the prior predictive under design $\tau$, and the $\theta^{(j)} \mathrm{s}$ are independent samples from the prior. To reduce cost and variance, we reuse the same $\theta$ and $\epsilon$ samples in all evaluations of $\hat{\mathcal{J}}_{\text {SIG }}(\tau)$. Following Overstall and Woods (2017) we take $B=B^{\prime}=1000$.

\section{J Realistic pharmacokinetic example}

Here we describe the analysis used for the realistic version of the pharmacokinetic example described in Section 7.5 of the main paper. Section J.1 describes the changes in methodology required, and Section J.2 gives further discussion of the results, which were presented in the main paper. 


\section{J.1 Methodology}

Gaps We wish to impose the constraint that observations must be separated by at least 0.25 hours. To do so it was sufficient to follow the approach described in Section 5.4 and simply add the following penalty term to our objective:

$$
\sum_{i=1}^{14} 1000 \max \left(0,0.25-\left[\tau_{(i+1)}-\tau_{(i)}\right]\right)
$$

where $\tau_{(i)}$ is the $i$ th observation time after sorting into increasing order. Thus a penalty is added whenever there are gaps between observation times less than 0.25 hours.

Multiplicative noise We also wish to use the multiplicative noise model (18) i.e.

$$
y_{i} \sim N\left(x\left(\theta, \tau_{i}\right), \sigma_{1}^{2}+\sigma_{2}^{2} x\left(\theta, \tau_{i}\right)^{2}\right) .
$$

This is a situation where the likelihood is available, so we use Algorithm 1 as described in Section G.2.

Let $\epsilon$ be a $N(0, I)$ random vector of length 30. Then observations from the multiplicative noise model can be expressed as

$$
y_{i}(\epsilon, \theta, \tau)=x\left(\theta, \tau_{i}\right)+\sigma_{1} \epsilon_{i}+\sigma_{2} x\left(\theta, \tau_{i}\right) \epsilon_{i+15},
$$

as required by assumption A6, and needed to implement Algorithm 1.

We found analyses using multiplicative noise take longer to converge. The likely explanation for this is that under the multiplicative noise the gradient estimates are more variable, since the Fisher information matrix must now be estimated. Therefore we use 500,000 iterations here, rather than the 100, 000 iterations used for the other pharmacokinetic analyses. These analyses took approximately half an hour to run, roughly 10 times as long as the analyses without multiplicative noise. Also, as discussed in Section G.2, in this time the multiplicative noise analyses perform only a single replication of experimental design, while the others perform 100 replications in parallel.

\section{J.2 Discussion}

Figure 6 in the main paper shows designs found from four analyses, under all combinations of with/without gaps and with/without multiplicative noise. The design with multiplicative noise and gaps is similar to those found in previous work using SIG: see Figure 2c in Overstall and Woods, 2017.

Close inspection of Figure 6 shows that the design points for multiplicative noise without gaps are slightly more spaced out than those without multiplicative noise. Inspection of trace plots suggest this is due to lack of full convergence of the optimisation algorithm. As mentioned above, this is likely due to more variable gradient estimates.

Each row of Figure 6 shows a design from a single run of a GDA optimisation algorithm. Therefore each gives a local optimum of our ADV objective (or a close 


\section{Bayesian experimental design without posterior calculations: supplement}

approximation to one). As in the other pharmacokinetic examples, we expect that there are multiple local optima which differ in the number of design points in each cluster. Selecting between these would require further work, such as applying point exchange when no gaps are used, or developing a similar post-processing method for the case with gaps.

\section{$\mathrm{K}$ Further details of geostatistical regression example}

Figure 6 shows trace plots of $\mathcal{J}_{\mathrm{ADV}}$ estimates, confirming convergence after 1000 iterations. It also shows that the improvement in this objective is largest for small $\ell$.
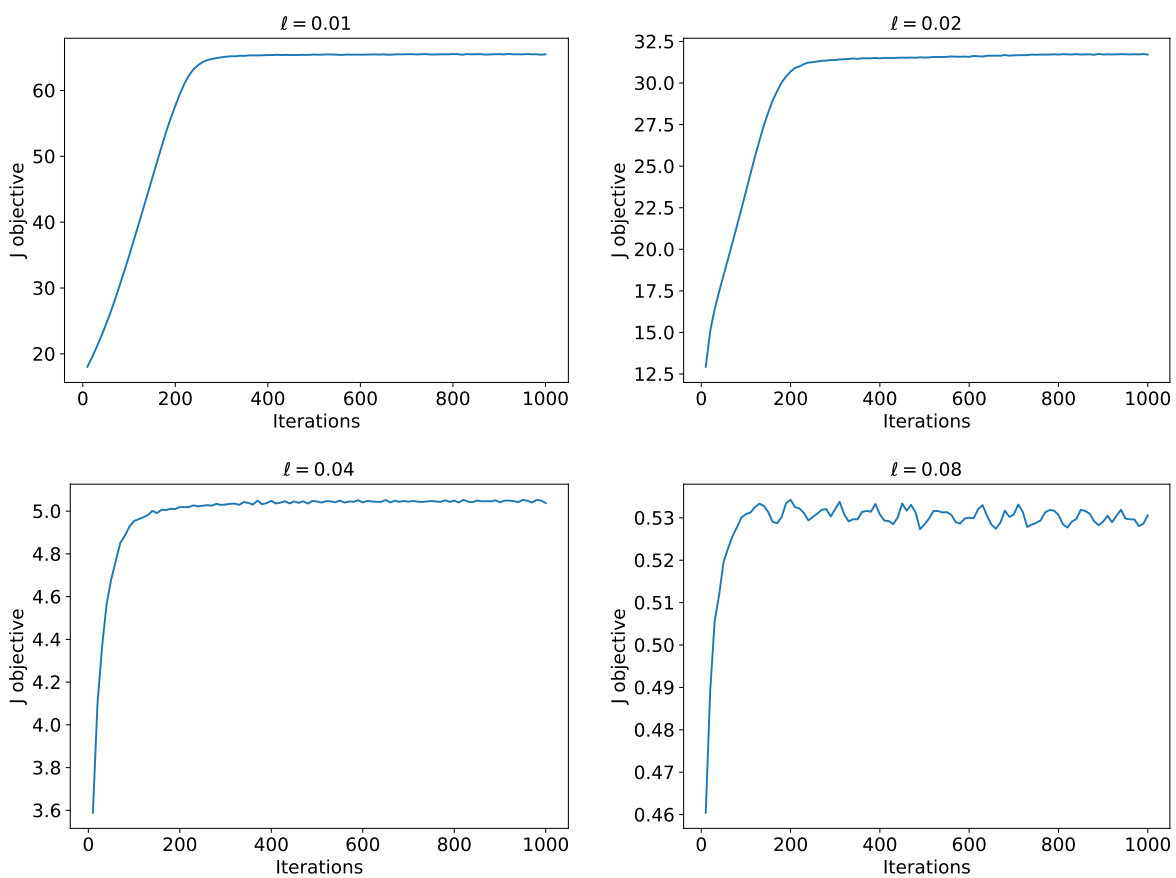

Figure 6: Trace plots of $\mathcal{J}_{\mathrm{ADV}}$ estimates for the geostatistical regression example.

\section{References}

Atkinson, A., Donev, A., and Tobias, R. (2007). Optimum experimental designs, with $S A S$. Oxford University Press. 7

Bernardo, J. M. (1979). Expected information as expected utility. The Annals of Statistics, 7(3):686-690. 2

Bradbury, J., Frostig, R., Hawkins, P., Johnson, M. J., Leary, C., Maclaurin, D., and 
Wanderman-Milne, S. (2018). JAX: composable transformations of Python+NumPy programs. http://github.com/google/jax. 10

Cappé, O., Moulines, E., and Rydén, T. (2006). Inference in hidden Markov models. Springer Science \& Business Media. 11

Cover, T. M. and Thomas, J. A. (2012). Elements of information theory. John Wiley \& Sons. 14, 20

Figurnov, M., Mohamed, S., and Mnih, A. (2018). Implicit reparameterization gradients. In Advances in Neural Information Processing Systems. 10

Foster, A., Jankowiak, M., Bingham, E., Horsfall, P., Teh, Y. W., Rainforth, T., and Goodman, N. (2019). Variational Bayesian optimal experimental design. In Advances in Neural Information Processing Systems. 11

Foster, A., Jankowiak, M., O'Meara, M., Teh, Y. W., and Rainforth, T. (2020). A unified stochastic gradient approach to designing Bayesian-optimal experiments. In International Conference on Artificial Intelligence and Statistics. 11, 16, 17, 18

Hyvärinen, A. (2005). Estimation of non-normalized statistical models by score matching. Journal of Machine Learning Research, 6:695-709. 1

Jankowiak, M. and Obermeyer, F. (2018). Pathwise derivatives beyond the reparameterization trick. In International Conference on Machine Learning. 10

Kleinegesse, S. and Gutmann, M. U. (2020). Bayesian experimental design for implicit models by mutual information neural estimation. In International Conference on Machine Learning. 11

Krause, A. and Guestrin, C. E. (2005). Near-optimal nonmyopic value of information in graphical models. In Uncertainty in Artificial Intelligence. 14

Maddison, C. J., Mnih, A., and Teh, Y. W. (2017). The concrete distribution: A continuous relaxation of discrete random variables. International Conference on Learning Representations. 10

Mohamed, S., Rosca, M., Figurnov, M., and Mnih, A. (2020). Monte Carlo gradient estimation in machine learning. Journal of Machine Learning Research, 21(132):1-62. 9

Overstall, A., Woods, D., and Adamou, M. (2019). acebayes: An R package for Bayesian optimal design of experiments via approximate coordinate exchange. Journal of Statistical Software. 16

Overstall, A. M. and Woods, D. C. (2017). Bayesian design of experiments using approximate coordinate exchange. Technometrics, 59(4):458-470. 7, 16, 17, 20, 21

Papamakarios, G., Nalisnick, E., Rezende, D. J., Mohamed, S., and Lakshminarayanan, B. (2021). Normalizing flows for probabilistic modeling and inference. Journal of Machine Learning Research, 22(57):1-64. 11

Poyiadjis, G., Doucet, A., and Singh, S. S. (2011). Particle approximations of the score 
24 Bayesian experimental design without posterior calculations: supplement and observed information matrix in state space models with application to parameter estimation. Biometrika, 98(1):65-80. 11

Shewry, M. C. and Wynn, H. P. (1987). Maximum entropy sampling. Journal of applied statistics, 14(2):165-170. 2 